\title{
On the nature of rainfall intermittency as revealed by different metrics and sampling approaches
}

\author{
G. Mascaro ${ }^{1,2}$, R. Deidda ${ }^{1}$, and M. Hellies ${ }^{1}$ \\ ${ }^{1}$ Universitá di Cagliari, Dipartimento di Ingegneria Civile, Ambientale e Architettura, Cagliari, Italy \\ ${ }^{2}$ Arizona State University, School of Sustainable Engineering and the Built Environment, AZ, USA
}

Correspondence to: G. Mascaro (gmascaro@unica.it)

Received: 7 August 2012 - Published in Hydrol. Earth Syst. Sci. Discuss.: 3 September 2012

Revised: 12 December 2012 - Accepted: 24 December 2012 - Published: 29 January 2013

\begin{abstract}
A general consensus on the concept of rainfall intermittency has not yet been reached, and intermittency is often attributed to different aspects of rainfall variability, including the fragmentation of the rainfall support (i.e., the alternation of wet and dry intervals) and the strength of intensity fluctuations and bursts. To explore these different aspects, a systematic analysis of rainfall intermittency properties in the time domain is presented using high-resolution (1-min) data recorded by a network of 201 tipping-bucket gauges covering the entire island of Sardinia (Italy). Four techniques, including spectral and scale invariance analysis, and computation of clustering and intermittency exponents, are applied to quantify the contribution of the alternation of dry and wet intervals (i.e., the rainfall support fragmentation), and the fluctuations of intensity amplitudes, to the overall intermittency of the rainfall process. The presence of three ranges of scaling regimes between $1 \mathrm{~min}$ to $\sim 45$ days is first demonstrated. In accordance with past studies, these regimes can be associated with a range dominated by single storms, a regime typical of frontal systems, and a transition zone. The positions of the breaking points separating these regimes change with the applied technique, suggesting that different tools explain different aspects of rainfall variability. Results indicate that the intermittency properties of rainfall support are fairly similar across the island, while metrics related to rainfall intensity fluctuations are characterized by significant spatial variability, implying that the local climate has a significant effect on the amplitude of rainfall fluctuations and minimal influence on the process of rainfall occurrence. In addition, for each analysis tool, evidence is shown of spatial patterns of the scaling exponents computed in the range of frontal systems. These patterns resem-
\end{abstract}

ble the main pluviometric regimes observed on the island and, thus, can be associated with the corresponding synoptic circulation patterns. Last but not least, we demonstrate how the methodology adopted to sample the rainfall signal from the records of the tipping instants can significantly affect the intermittency analysis, especially at smaller scales. The multifractal scale invariance analysis is the only tool that is insensitive to the sampling approach. Results of this work may be useful to improve the calibration of stochastic algorithms used to downscale coarse rainfall predictions of climate and weather forecasting models, as well as the parameterization of intensity-duration-frequency curves, adopted for land planning and design of civil infrastructures.

\section{Introduction}

The investigation of rainfall statistical variability is of paramount importance given the central role of this geophysical variable in a wide range of disciplines, including hydrology (Georgakakos and Kavvas, 1987; Dingman, 2008), meteorology (Huffman et al., 1997; Trenberth et al., 2003), hydrometeorology (Seo et al., 2000; Langousis and Veneziano, 2009a,b; Cuo et al., 2011), ecology (Eagleson, 2002), and agronomy (Moonen et al., 2002). A considerable number of studies have focused on the characterization and simulation of rainfall intermittency in the time domain, a concept often used to refer to two diverse aspects of variability: (i) the alternation of dry and wet periods, identified through the construction of the binary series, which form the so-called support of the measure (Verrier et al., 2011; Kundu and Siddani, 2011; Schleiss et al., 2011); and (ii) the sudden variations of 
the rainfall intensity occurring over the support (Lovejoy and Schertzer, 1990; Venugopal et al., 1999; Deidda, 2000; Deidda et al., 2004, 2006; Molini et al., 2009; Rigby and Porporato, 2010). Rainfall intermittency can thus be attributed to two components: the variability of the support and, for a given support, the fluctuations of the amplitudes of rainfall intensity (Veneziano and Langousis, 2005a,b; Langousis and Veneziano, 2007; Veneziano et al., 2006, 2007).

The increasing availability of large records of highresolution (up to few tens of seconds) point measurements provided by automatic rain gauges and, in some recent experiments, by disdrometers has allowed the study of intermittency properties in the time domain within a wide range of scales. For this purpose, techniques originally adopted to examine scalar turbulence have been used, including spectral analysis (Rebora et al., 2006), investigation of scale invariance and multifractality (Veneziano et al., 2006, and references therein), and wavelet-based methods (Venugopal et al., 2006). The application of such tools has revealed the existence of different scaling regimes, i.e., time intervals where the rainfall statistical properties can be expressed through power law relations across scales (Fraedrich and Larnder, 1993; Deidda et al., 1999; Verrier et al., 2011). A correspondence has often been found between these regimes and the typical duration of weather phenomena (e.g., Fraedrich and Larnder, 1993). In a recent study, Molini et al. (2009) combined these techniques with the computation of clustering and intermittency exponents (Bershadskii et al., 2004; Sreenivasan and Bershadskii, 2006), aiming at weighting the contribution of the alternation of dry and wet phases to the overall intermittency of rainfall signals. These authors used data observed by five rain gauges located in different climates and found that the scaling and intermittency properties have distinct features across gauges (i.e., climatic conditions), whereas the support variability has similar characteristics.

The analysis of rainfall intermittency properties has been fundamental to develop stochastic models reproducing rainfall time series at multiple scales, which are useful for multiple hydrological applications, including flood and flashflood forecasting (Mascaro et al., 2010; Rigby and Porporato, 2010), evaluation of water resources availability (Burton et al., 2010), and design of civil infrastructures (Veneziano et al., 2007). These models range from those based on the fractal and multifractal theories (Lovejoy and Schertzer, 1985; Veneziano et al., 1996; Deidda, 2000; Langousis et al., 2009), to algorithms assuming parametric distributions of storm occurrence and structure (i.e., number, intensity and duration of individual cells) (Rodriguez-Iturbe et al., 1987; Onof and Wheater, 1993; Robinson and Sivapalan, 1997).

Most studies on rainfall intermittency analyzed time series collected by a small number of gauges or disdrometers (e.g., Deidda et al., 1999; Molini et al., 2009; Verrier et al., 2011) with the drawback that the statistical significance of their results is somehow limited: no information on the spatial variability of these statistical properties within regional domains can be derived, and the identification of the possible linkages with local terrain characteristics and dominant synoptic circulation becomes problematic. In this paper, we attempt to overcome these limitations by characterizing intermittency of rainfall intensity and support on the island of Sardinia (Italy) using time series collected by 201 tipping-bucket gauges, with tipping accuracy of $0.2 \mathrm{~mm}$ of rainfall depth at time precision of $1 \mathrm{~s}$. Specifically, we pursue the following main objectives. First, we apply several techniques to investigate the intermittency properties of the rainfall time series recorded at each station aiming at the following: (a) assessing the effectiveness of each technique to characterize diverse aspects of rainfall intermittency; (b) identifying the presence of multiple scaling regimes and computing, for each of them, a number of metrics that permit intermittency quantification related to the fluctuations of rainfall intensity and the fragmentation or clusterization of its support; and (c) investigating the relative contribution to the intermittency properties due to rainfall intensity fluctuations and support fragmentation. Second, for each scaling regime, we explore the possible existence of spatial patterns for the metrics and we search for linkages with the dominant synoptic circulations that affect the pluviometric regimes of the island and the topographic features of the gauge location.

Finally, we focus on a third objective related to the effect of the sampling methodology (used to build the rainfall intensity signal) on the intermittency analysis. Specifically, we focus on the tipping-bucket effect (or quantization), which is related to the method usually adopted to derive the rainfall intensity series from the tipping instants (e.g., if the volume of each bucket is equivalent to $0.2 \mathrm{~mm}$ rainfall depth and the time resolution is $1 \mathrm{~min}$, this will lead to a discretized signal with records multiple of $12 \mathrm{~mm} \mathrm{~h}^{-1}$ ). The importance of the tipping-bucket effect and other instrumental artifacts in the estimation of some intermittency metrics (namely, power spectra slopes and moment scaling exponents) has been investigated on synthetical time series by Harris et al. (1997) and Veneziano and Furcolo (2009). Using an observed rainfall time series at 15 -min resolution derived by digitizing pluviographs of a float-and-syphon-type gauge, de Lima and Grasman (1999) showed that the procedure used to sample the signal and the gauge characteristics affects the multifractal analysis, especially when considering statistics related to the lowest and the highest rainfall intensities. Here, we systematically investigate the tipping-bucket effects when estimating some intermittency metrics on the wide rainfall database described above and we suggest an alternative methodology that allows building the signal in a more physically realistic fashion, circumventing quantization artifacts. 


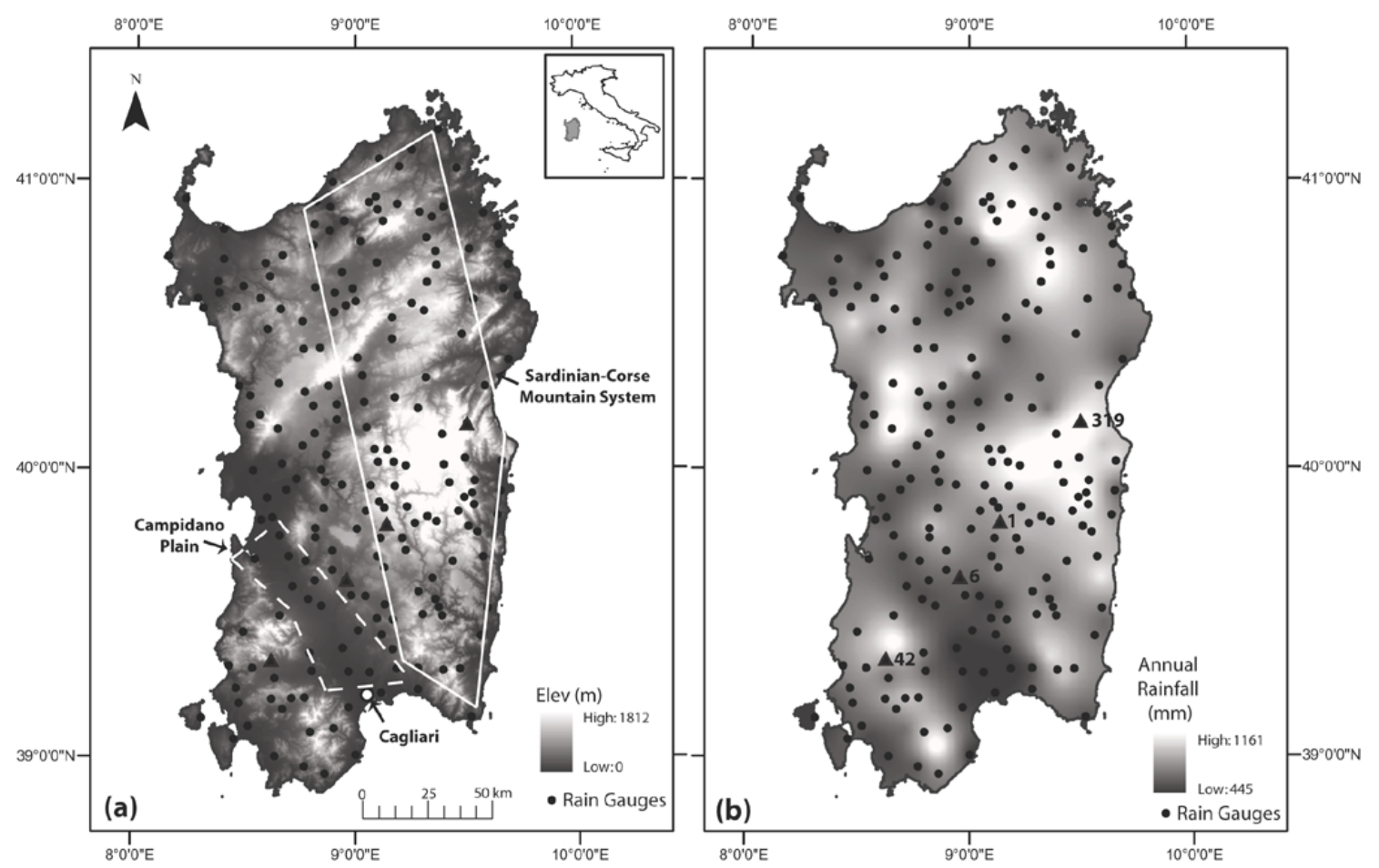

Fig. 1. (a) Digital elevation model of Sardinia (Italy). The inset shows the geographic location of the island with respect to the Italian peninsula. The polygons delimited by dashed and continuous white lines are the approximate boundaries of the Campidano plain and of the Sardinian-Corse Mountain System, respectively. (b) Spatial distribution of the annual rainfall. In both panels, the black circles indicate the location of the 201 stations used for the analyses, while the triangles identify the gauges with IDs 1, 6, 42 and 319 adopted as representative cases for the subsequent analyses.

\section{Study area and pluviometric regimes}

The study area is Sardinia (Fig. 1), an island of $\sim 24000 \mathrm{~km}^{2}$ located in the Mediterranean Sea, about $400 \mathrm{~km}$ west off of the Italian peninsula (inset in Fig. 1a). Apart from the Campidano plain (dashed white polygon in Fig. 1a) and a small area in the northwestern corner, topography is rather complex, as shown in the digital elevation model (DEM) reported in Fig. 1a. A long mountain range, called Sardinian-Corse Mountain System (white polygon in Fig. 1a), is located in the eastern part of the island, running from north to south. In addition, a smaller isolated mountain range is located in the southwest.

The climate of Sardinia is Mediterranean with extremely dry summers and rainfall falling for the greatest part during the period from September to May. The spatial distribution of annual rainfalls is shown in Fig. 1b, produced by applying the kriging technique to annual rainfall averages obtained from 70 -yr-long rainfall records collected by 201 rain gauges operating at daily resolution. No information about the elevation was included to interpolate rainfall through kriging. Comparison with Fig. 1a clearly reveals a strong relation between the annual rainfall depth and elevation: in areas of lower elevation, the total rainfall is about $500 \mathrm{~mm}$ per year, reaching $1160 \mathrm{~mm}$ at the highest mountains.
Chessa et al. (1999) applied different cluster analysis techniques to study the winter (from September to May) rainfall regimes over Sardinia and the linkages with synoptic circulation. The analysis was performed using daily rainfall depths from 114 gauges and spatial fields of meteorological variables at $5^{\circ}$ resolution, provided by the National Center for Atmospheric Research (NCAR) analysis. These authors identified three main clusters of rainfall spatial patterns on the island, reported in Fig. 2, associated with different dominant synoptic conditions. Clusters 1 and 2 are characterized by a limited negative gradient of rainfall intensity from SW to NE (cluster 1) and from NW to SE (cluster 2). In both cases, the Sardinian-Corse Mountain System leads to lower precipitation amount in the eastern part of the island, and the dominant synoptic patterns are characterized by northwesterly flows (the mistral wind) bringing large frontal systems.

Cluster 3 is completely different and is characterized by a strong $\mathrm{E}-\mathrm{W}$ negative rainfall gradient, with synoptic circulation associated with Atlantic flow passing over Northern Africa and crossing the southern part of the Mediterranean Sea. Under these conditions, moist air at lower levels of the atmosphere is transported towards Sardinia by southeasterly winds (the sirocco wind) while, simultaneously, cold air arrives at upper levels from the north. This potential instability state is further enhanced by the orographic barrier 
in the eastern part of the island and by the mountain ranges in the south (Fig. 1a). Under this type of synoptic condition, precipitation events of high intensity (frequently on the order of $300 \mathrm{~mm}$ and sometimes of more than $500 \mathrm{~mm}$ accumulated in $24 \mathrm{~h}$, with peaks exceeding $100 \mathrm{~mm}$ in less than $1 \mathrm{~h}$ ) have been observed, especially during the autumn season when the sea temperature is relatively high. These storms have caused severe floods in the territories located along the eastern coast of the island and close to Cagliari (the main town), with significant property damage and loss of lives (Chessa et al., 2004).

\section{Dataset and construction of rainfall signals}

We used rainfall time series collected by a total of 201 rain gauges covering the entire island with approximately uniform density in terms of both space and elevation (black dots in Fig. 1a and b). The pluviometers belong to the network of the Sardinian Hydrological Survey and are all of the tippingbucket-type, with an accumulated rain depth of $0.2 \mathrm{~mm}$ per tip and an electronic apparatus that records the tipping instants in a digital memory with a time precision of $1 \mathrm{~s}$. The high-resolution dataset spans over $11 \mathrm{yr}$ of observations from 1986 to 1996, with periods of instrument malfunction of various lengths in each station. This dataset was previously utilized by Badas et al. (2006) to test an approach for including the effect of orography in the calibration of a multifractal model in a spatiotemporal framework.

Most of the time series of rainfall observations collected by tipping-bucket rain gauges are usually provided as rainfall intensity $i_{\Delta t}$ at a given resolution $\Delta t$ derived by (i) computing the accumulated rain depth within each step $\Delta t$ by multiplying $0.2 \mathrm{~mm}$ (or the rainfall depth corresponding to the bucket volume) by the number of tips occurred in $\Delta t$, and (ii) dividing the resulting rain depth by $\Delta t$. Hence, this approach assumes that (i) the rainfall depth needed to fill one bucket (e.g., $0.2 \mathrm{~mm}$ ) is attributed to the current time step (which is equivalent to assuming that the rain entirely falls when the tip is recorded), and (ii) the relation between cumulative rainfall depth and time is a step-wise line. The resulting signal is thus discretized (the method will be hereafter labeled as DC, discrete counting). The use of DC leads to two disadvantages that potentially affect the intermittency analysis, especially for low $\Delta t$. First, when only one tip occurs within a time step $\Delta t$, the method returns a relatively high intensity that may not have physical sense. For example, the World Meteorological Organization (WMO) suggests assuming a resolution of $\Delta t=1 \mathrm{~min}$ (see, e.g., Lanza et al., 2005, and references therein). Hence, the minimum non-zero intensity is $0.2 \mathrm{~mm} / 1 \mathrm{~min}=12 \mathrm{~mm} \mathrm{~h}^{-1}$. Second, it is possible that $i_{\Delta t}=0$ is assigned to time steps included within a continuous (i.e., without interruptions) rainfall event characterized by low intensity. To overcome the drawbacks of DC and to investigate the effects that the use of this method causes when
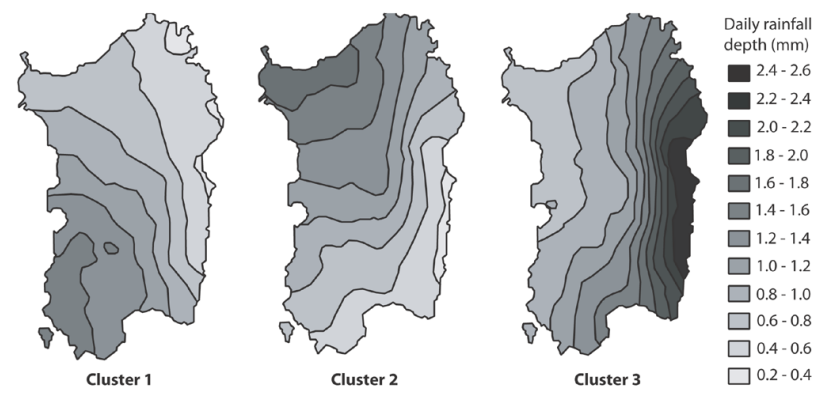

Fig. 2. Main classes of rainfall spatial patterns in the Sardinian region (adapted from Chessa et al. (1999)); see main text for details.

studying rainfall intermittency, here we suggest an alternative approach, based on the hypothesis that the relation between cumulative rainfall depth and time is obtained through linear interpolation between the tipping instants. This method (labeled as CC, continuous counting) is described in detail in Appendix A together with the DC strategy. As a matter of fact, the $\mathrm{CC}$ approach allows the signal to be sampled in a more realistic manner.

The dataset used in this study was built by sampling the signals of the tipping instants with both CC and DC methods at a resolution $\Delta t=1 \mathrm{~min}$. For each gauge, we extracted continuous time sequences with duration $T=2^{16}$ ( $\sim 45$ days). The values selected for $\Delta t$ and $T$ permit investigating intermittency in a range of scales of great interest for hydrological applications. Despite the presence of missing data, we were able to extract a minimum of 29 events (i.e., about $3.5 \mathrm{yr}$ of data) per station with variable mean intensity, including some zero values observed in the summer. For each gauge, analyses were performed on the quartile of events with the highest mean intensity resulting in a mean, minimum and maximum number of 12,7 , and 17 events per pluviometer, respectively. In addition to the rain intensity signal $i_{\Delta t}$ (hereafter FS, full signal), we created the binary transformation (hereafter BS, binary signal) to study the intermittency associated with the support. This is simply defined as $\operatorname{BS}\left(i_{\Delta t}\right)=1$ if $i_{\Delta t}>0$, or $\operatorname{BS}\left(i_{\Delta t}\right)=0$ if $i_{\Delta t}=0$.

\section{Methods}

In this section, we describe the techniques used to characterize the intermittency properties of rainfall time series, including the classical tools of spectral and scale invariance analysis, and the clustering and intermittency exponents. Each tool involves the investigation of a scaling law with a corresponding exponent that was utilized as a metric to quantify intermittency of the full and the binary series (FS and BS).

\subsection{Spectral and (multifractal) scale invariance analysis}

We used the fast Fourier transform to compute the power spectra of FS and BS in the range of scales included between 

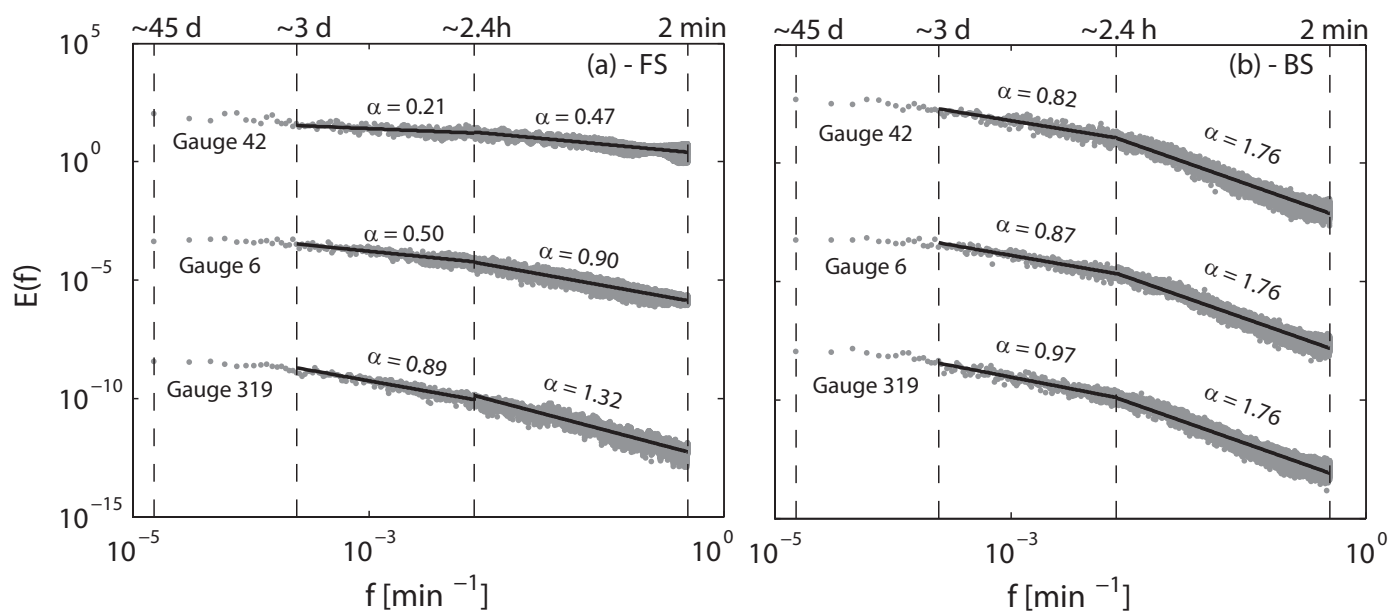

Fig. 3. Example of scaling regimes obtained through the spectral analysis for gauges with IDs 42, 6 and 319 (shown in Fig. 1b), for (a) the FS and (b) the BS series. The slopes of the lines are estimates of the spectral exponents $\alpha^{\mathrm{FS}}$ and $\alpha^{\mathrm{BS}}$ in Eq. (1). Arbitrary units on the y-axis are used in order to display results from different gauges in the same graph.

Table 1. Summary of the metrics used to investigate rainfall intermittency, including symbol, description, reference to the equation of the corresponding scaling law, and symbols adopted in the application to the full and/or the binary series (FS and BS, respectively).

\begin{tabular}{llcll}
\hline Symbol & Description & Equation & FS & BS \\
\hline$\alpha$ & Spectral Exponent & $(1)$ & $\alpha^{\mathrm{FS}}$ & $\alpha^{\mathrm{BS}}$ \\
$K(q)$ & Multifractal Exponent & $(3)$ & $K(q)$ & - \\
$\phi$ & Clustering Exponent & $(4)$ & - & $\phi$ \\
$\mu_{2}$ & Intermittency Exponent & $(6)$ & $\mu_{2}^{\mathrm{FS}}$ & $\mu_{2}^{\mathrm{BS}}$ \\
\hline
\end{tabular}

$2^{16} \min (\sim 45$ days $)$ to 2 min. For a given gauge, a single spectrum was produced by averaging, for each frequency $f$, the energy $E(f)$ of all available $\sim 45$-day sequences. Following evidence of previous studies (e.g., Fraedrich and Larnder, 1993; Verrier et al., 2011), we investigated the presence of characteristic ranges of scaling regimes through the following power law:

$E(f) \sim f^{-\alpha}$,

where $\alpha$ is the spectral exponent that can be estimated via linear regression by applying the logarithms on both sides of Eq. (1). Hence, for each gauge and range of scaling regime, two spectral exponents for FS and BS were estimated and labeled as $\alpha^{\mathrm{FS}}$ and $\alpha^{\mathrm{BS}}$.

The multifractal scale invariance analysis was carried out only on the FS time sequences over scales ranging from a fine $\left(\tau_{0}\right)$ to a coarse $\left(T=\tau_{0} \cdot 2^{M}\right.$, where $M$ is a non-negative integer) scale and, then, computing the partition function (see, e.g., Deidda et al., 1999):

$S_{q}(\tau)=\frac{1}{N(\tau)} \sum_{k=1}^{N(\tau)}\left[i_{\tau, k}\right]^{q}$, where $i_{\tau, k}$ is the rainfall intensity aggregated at scale $\tau=\tau_{0} \cdot 2^{j}(j=0, \ldots, M)$ in the $k$-th time step, $N(\tau)$ the number of non-overlapping steps $\tau$ included in $T$, and $q$ the considered moments. Hence, for a given $q, S_{q}(\tau)$ is calculated for the different aggregation scales $\tau$ and the presence of scale invariance is investigated by verifying that the power law holds for different $q$ :

$S_{q}(\tau) \sim \tau^{-K(q)}$.

This is accomplished again by plotting Eq. (3) in the loglog plane and estimating the slope $K(q)$ through linear regression. The series is multifractal (fractal) if the exponents $K(q)$ are non-linearly (linearly) related to $q$. Here, the scale invariance analysis was carried out from $\tau_{0}=\Delta t=1 \mathrm{~min}$ to $T=2^{16} \min (\sim 45$ days $)$ and for the moments $q=1.5,2,2.5$ and 3. For a given station, the analysis was conducted on a single set of mean partition functions, computed by averaging the $S_{q}(\tau)$ of all available $\sim 45$-day time sequences.

\subsection{Clustering and intermittency exponents}

The clustering exponent is a metric defined only for BS. It was proposed to analyze turbulence fluxes by Sreenivasan and Bershadskii (2006) and adapted to investigate the rainfall intermittency component due to the support of the measure by Molini et al. (2009). The clustering exponent is computed as follows. Each BS $\left(i_{\Delta t}\right)$ series with resolution $\Delta t$ and length $T=\Delta t \cdot 2^{M}$ (where, again, $M$ is a non-negative integer) is divided in consecutive, non-overlapping time windows $\tau=\Delta t \cdot 2^{j}$, with scale index $j=1, \ldots,(M-1)$. The number $N$ of zero crossing (i.e., the transition from rain to no-rain and viceversa) of $\mathrm{BS}\left(i_{\Delta t}\right)$ within each window $\tau$ is counted, and the corresponding rate $n(\tau)=N / \tau$ is derived. Next, the standard deviation $\left\langle[\delta n(\tau)]^{2}\right\rangle^{1 / 2}$ of the fluctuations $\delta n(\tau)=n(\tau)-\langle n(\tau)\rangle$ is computed, where $\langle\cdot\rangle$ is the average 
Table 2. Mean and standard deviation (in parentheses) across the 201 gauges of the metrics in the different scaling regimes (separated by square brackets). The breaking points of the time ranges for each metric are reported in the header, where we also indicate the characteristics of the weather phenomena used to interpret the spectra scaling regimes by Fraedrich and Larnder (1993). The metrics have been calculated on FS and/or BS signals constructed with the CC method.

\begin{tabular}{|c|c|c|c|c|c|c|c|c|c|c|}
\hline & {[} & \multicolumn{3}{|c|}{ Storms } & ][ & Frontal Systems & ][ & \multicolumn{2}{|c|}{ Transition } & ] \\
\hline & 1 & 2 & & $2^{5}$ & $2^{7}$ & & $2^{12}$ & $2^{14}$ & $2^{15}$ & $2^{16}$ \\
\hline & $1 \mathrm{~min}$ & $2 \mathrm{mir}$ & & $32 \mathrm{~min}$ & $\sim 2.4 \mathrm{~h}$ & & $\sim 3 \mathrm{~d}$ & $\sim 11 \mathrm{~d}$ & $\sim 22 \mathrm{~d}$ & $\sim 45 \mathrm{~d}$ \\
\hline$\alpha^{\mathrm{FS}}$ & & {[} & \multicolumn{2}{|c|}{$1.12(0.35)$} & ][ & $0.55(0.18)$ & ][ & \multicolumn{2}{|c|}{ Not Computed } & ] \\
\hline$\alpha^{\mathrm{BS}}$ & & {[} & \multicolumn{2}{|c|}{$1.76(0.02)$} & ][ & $0.92(0.07)$ & ][ & \multicolumn{2}{|c|}{ Not Computed } & ] \\
\hline$K(3)$ on FS & {[} & \multicolumn{2}{|c|}{$0.77(0.30)$} & ][ & & $1.36(0.14)$ & & ][ & $1.04(0.18)$ & ] \\
\hline$\phi$ on BS & & [ & $0.45(0.01)$ & ][ & & $27(0.02)$ & ][ & $0.64(0.10)$ & ] & \\
\hline$\mu_{2}^{\mathrm{FS}}$ & & {[} & $0.74(0.07)$ & ][ & & $86(0.06)$ & ][ & $0.70(0.07)$ & ] & \\
\hline$\mu_{2}^{\mathrm{BS}}$ & & [ & $0.88(0.02)$ & ][ & & $49(0.04)$ & ][ & $0.41(0.05)$ & ] & \\
\hline
\end{tabular}

operator over all windows of size $\tau$. The procedure is repeated for different scales of observation $\tau$, and the presence of ranges of scaling regimes is investigated through the relation:

$\left\langle[\delta n(\tau)]^{2}\right\rangle^{1 / 2} \sim \tau^{-\phi}$,

where the exponent $\phi$ of the power law is the clustering exponent. In this study, the scaling relation Eq. (4) was tested in the scale range from $2 \mathrm{~min}$ to $2^{15} \mathrm{~min}(\sim 22$ days $)$. For a given gauge, we computed $\left\langle[\delta n(\tau)]^{2}\right\rangle^{1 / 2}$ for all available $\sim 45$-day sequences and, for each $\tau$, we averaged the corresponding values. The relation Eq. (4) was then investigated using a single set of mean $\left\langle[\delta n(\tau)]^{2}\right\rangle^{1 / 2}$ per gauge.

The intermittency exponent is a metric computable for both FS and BS. Like the clustering exponent, it was originally adopted as a tool for turbulence investigation (Bershadskii et al., 2004) and applied to rainfall time series by Molini et al. (2009). To illustrate how the intermittency exponent is obtained, let us refer to the FS series $i_{\Delta t}$ (the application for BS is analogous). As for the multifractal scale invariance analysis, the time series is divided in consecutive, nonoverlapping windows $\tau=\Delta t \cdot 2^{j}(j=0, \ldots, M)$. For a given $\tau$, the variable

$\chi_{\tau}=\frac{1}{\tau} \sum_{k=1}^{N(\tau)}\left(\left|\frac{i_{\Delta t, k}-i_{\Delta t, k-1}}{\Delta t}\right|^{2} \Delta t\right)$

is computed within each window $\tau$. In Eq. (5), $N(\tau)$ is the total number of windows $\tau$ within the series of length $T$ and $\left(i_{\Delta t, k}-i_{\Delta t, k-1}\right) / \Delta t$ is the gradient of $i_{\Delta t}$ for the $k$-th time step. The metric $\chi_{\tau}$ provides an average measure of the signal variability and intermittency within each time window $\tau$. The procedure is repeated for different scales of observation $\tau$ and the presence of the scaling relation is tested:

$\frac{\left\langle\chi_{\tau}^{2}\right\rangle}{\left\langle\chi_{\tau}\right\rangle^{2}} \sim \tau^{-\mu_{2}}$.

In Eq. (6), $\mu_{2}$ is the intermittency exponent, estimated through linear regression in the log-log plane. For each sta- tion and range of scaling regime, two single values of the intermittency exponent were calculated, one for FS (indicated with $\left.\mu_{2}^{\mathrm{FS}}\right)$ and the other for BS $\left(\mu_{2}^{\mathrm{BS}}\right)$, following the same approach illustrated for the other exponents. We highlight that previous studies (Veneziano and Iacobellis, 1999; Neuman, 2010a,b, 2012; Guadagnini and Neuman, 2011) have demonstrated that techniques based on the gradient amplitude method, like the intermittency exponent, are not able to reveal presence of scaling and multifractal properties. Thus, the intermittency exponent was here only utilized to compare the intermittency characteristics of BS and FS series. For the sake of clarity, the metrics investigated in this study are summarized in Table 1.

\section{Results and discussion}

In this section we present and discuss the main results arising from the systematic application of the methods previously described to the rainfall time series observed in the 201 gauges. The section is divided into four subsections. In the first three, results are presented for the series built with the CC method: in Sect. 5.1, we show evidence of scaling regimes as emerged by applying each of the four metrics; in Sect. 5.2, we analyze and compare the intermittency properties of rainfall intensity and support, while in Sect. 5.3 we discuss the existence of spatial patterns for the metrics on the island and of linkages with topography and weather patterns. Finally, in the last Sect. 5.4 we compare results obtained with the $\mathrm{CC}$ method with those returned by analyzing the signals sampled with the discrete counting (DC) approach.

\subsection{Evidence of scaling regimes and summary of metrics}

The investigation of the scaling laws Eqs. (1), (3), (4), and (6) on the FS and/or BS series derived from the 201 gauges allowed the identification of three ranges of scaling regimes, with breaking points (i.e., the times separating the regimes) determined by applying the method described 

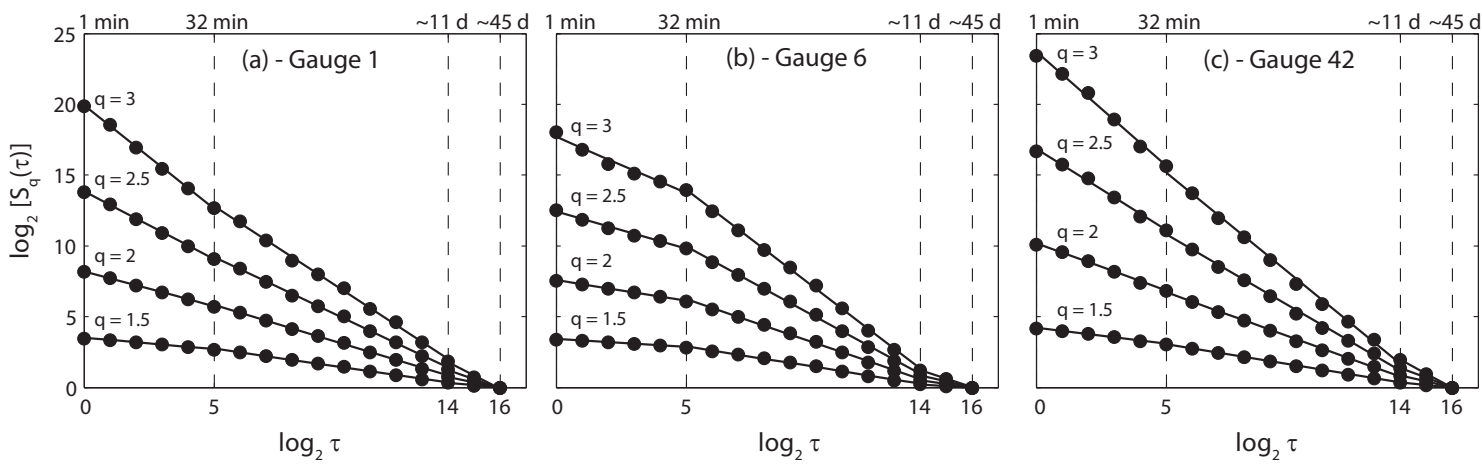

Fig. 4. Example of scale invariance regimes for gauges with IDs 1, 6 and 42 (shown in Fig. 1b) - subplots (a), (b) and (c), respectively. The slopes of the lines are estimates of the multifractal exponents $K(q)$ in Eq. (3).

in Appendix B. As discussed below in more detail, results of our analyses indicate the following: (i) for a given technique, the positions of the breaking points are fairly constant for most gauges, and (ii) the location of the breaking points changes with the metric, suggesting that these tools provide diverse information about the intermittency properties of the rainfall series.

The spectra for FS and BS have similar shape characterized by the presence of three scaling regimes according to Eq. (1): from $2^{16} \min \left(\sim 45\right.$ days) to $2^{12} \min (\sim 3$ days $)$ the spectra are almost flat, while two scaling laws with distinct spectral exponents emerge in the ranges from $2^{12} \mathrm{~min}$ to $2^{7} \mathrm{~min}(\sim 2 \mathrm{~h})$, and from $2^{7} \mathrm{~min}$ to $2 \mathrm{~min}$. Figure 3 show examples of the spectra computed for FS and BS for three gauges (IDs 42, 6 and 319) with contrasting behavior. The shape of the spectra and the numerical values of the exponents are similar to those reported in previous studies focused on diverse climatic settings and scale ranges from years to minutes (see, e.g., Verrier et al., 2011, and references therein). The existence of three scaling regimes was also found through the scale invariance analysis of Eq. (3), but the breaking points are located at $2^{14} \min (\sim 11$ days $)$ and $2^{5}(32)$ min. A similar discrepancy with the spectral analysis was also found by Verrier et al. (2011) and is likely due to the different sampling methods involved in these techniques to analyze the signal at diverse scales. Evidence of scale invariance in the time series observed in three gauges is shown in Fig. 4. We highlight that, in a small number of stations, the existence of a single regime from $\sim 11$ days to $1 \mathrm{~min}$ may be identified (e.g., gauge 42 in Fig. 4c). Finally, the computation of the clustering and intermittency exponents showed the presence of breaking points at $2^{12} \mathrm{~min}$ ( $\sim 3$ days), as found with the spectral analysis, and at $2^{5}$ (32) min, as emerged through the investigation of scale invariance. Figures 5 and 6 show examples of outcomes of these analyses for three stations.

To summarize the results, in Table 2 we reported the breaking points separating the scaling regimes identified by each analysis and, for a given regime, the mean and the standard deviation across the gauges of all metrics, including the spectral exponents of FS and BS ( $\alpha^{\mathrm{FS}}$ and $\alpha^{\mathrm{BS}}$, respectively), the multifractal exponents $K(3)$ for moment $q=3$, the clustering exponent $\phi$ and the intermittency exponents for FS and $\mathrm{BS}$ ( $\mu_{2}^{\mathrm{FS}}$ and $\mu_{2}^{\mathrm{BS}}$, respectively). In addition, we indicated the kind of meteorological phenomena typical of each time regime. For this purpose, we referred to the definition provided by Fraedrich and Larnder (1993) to interpret the spectrum: the flat portion of the spectrum at scales larger than $\sim 3 \mathrm{~d}$ is characteristic of a transition zone; the range from $\sim 3 \mathrm{~d}$ to $\sim 2.4 \mathrm{~h}$ is typical of frontal systems; and the interval from $\sim 2.4 \mathrm{~h}$ to $2 \mathrm{~min}$ is dominated by convective storms or single rainfall cells.

\subsection{Intermittency properties of rainfall intensity and support}

Examination of Table 2 allows drawing some important considerations on the intermittency features of rainfall intensity and support signals. First, we highlight that the standard deviations of the metrics characterizing $\mathrm{BS}\left(\alpha^{\mathrm{BS}}, \phi\right.$, and $\left.\mu_{2}^{\mathrm{BS}}\right)$ are much lower than those of the metrics calculated on FS $\left(\alpha^{\mathrm{FS}}, K(3)\right.$, and $\left.\mu_{2}^{\mathrm{FS}}\right)$. This implies that, while the statistical properties of the rainfall intensity significantly vary within our regional study site, the corresponding support has instead very similar intermittency characteristics across the island. Hence, the local climate has minimal influence on the support fragmentation and a stronger effect on the variability of the rainfall intensity. This result is in accordance with findings of Molini et al. (2009) obtained with series observed in different climatic settings.

The values obtained for the spectral exponents $\alpha^{\mathrm{FS}}$ and $\alpha^{\mathrm{BS}}$ are higher in the scaling regime typical of single storms, both for FS and BS series. Higher spectral exponents imply faster decay of the spectra energy from larger to smaller temporal scales, indicating the existence of individual rainfall cells localized in time (e.g., convective storms) (Purdy et al., 2001). In contrast, lower values of $\alpha^{\mathrm{FS}}$ and $\alpha^{\mathrm{BS}}$ are found in the scaling regime typical of frontal systems: in this 


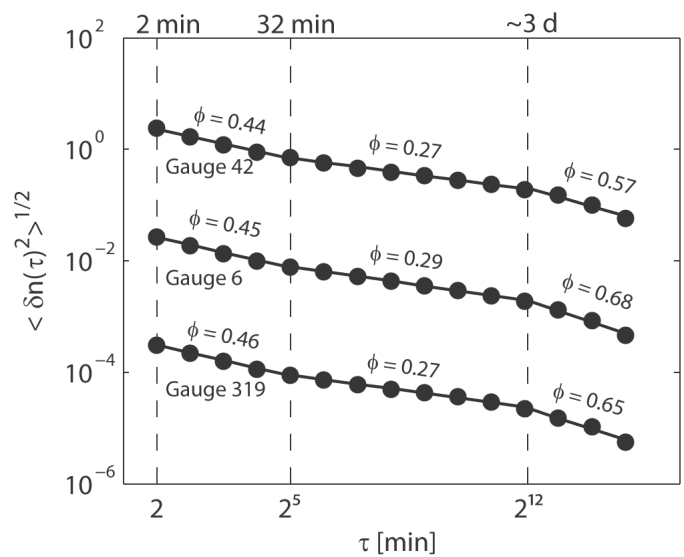

Fig. 5. Example of scaling regimes using the clustering exponent $\phi$ for gauges with IDs 42, 6, and 319 (shown in Fig. 1b). The slopes of the lines are estimates of $\phi$ in Eq. (4). Arbitrary units on the yaxis are used in order to display results from different gauges in the same graph.

case, the energy decreases with a smaller rate from larger to smaller time scales, consistent with the presence of longer, stratiform rainfall systems (Purdy et al., 2001).

The multifractal scale invariance analysis accounts for another characteristic of rainfall intermittency, which is the rate of dissipation of the fluctuations of FS as a function of the aggregation scale. A high $K(3)$ is obtained when the signal is more variable and intermittent, with presence of uneven peaks, when it is sampled at smaller scales. These sudden variations are then smoothed out as the scale of aggregation increases (i.e., the partition function $S_{3}(\tau)$ decreases faster for increasing $\tau$ ). In contrast, low values of $K(3)$ refer to smooth signals with small fluctuations across the aggregation scales (the extreme case of a uniform signal is characterized by $K(3)=0$ ). Table 2 shows that $K(3)$ is the highest in the range dominated by frontal systems (1.36), is characterized by intermediate values in the transition zone (1.04), and receives the smallest values in the storm regime (0.77). We highlight that the intermittency exponent of FS, $\mu_{2}^{\mathrm{FS}}$, provides similar information to the scale invariance analysis, because the computation of the intermittency exponent is based on the signal gradients (Eq. 5) and is thus directly affected by the rainfall intensity fluctuations.

The clustering exponent $\phi$ has an average value of 0.45 in the range from 32 to $2 \mathrm{~min}$ associated with convective storms and single rain cells. This is close to 0.5 , which is the expected value for the white noise (Sreenivasan and Bershadskii, 2006), indicating low clustering and high randomness of the process. The mean $\phi$ decreases to 0.27 (i.e., higher clustering of dry and wet periods) in the range from $\sim 3$ days to 32 min, likely because of the larger compactness of the frontal systems typical of these scales. In the transition zone, the average $\phi$ is 0.64 , higher than the expected value for the
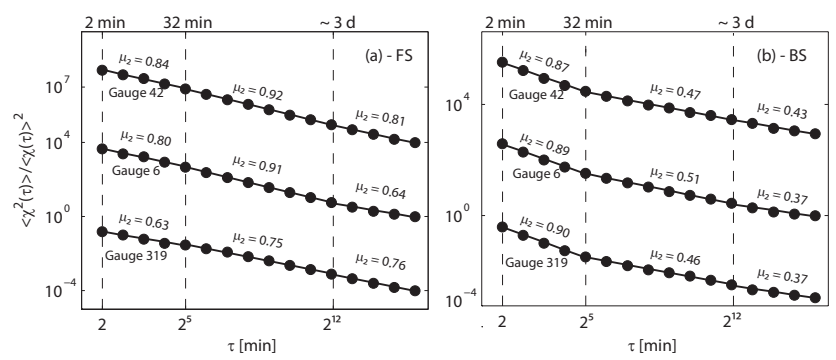

Fig. 6. Example of scaling regimes obtained by computing the intermittency exponent $\mu_{2}$ for gauges with IDs 42, 6, and 319 (shown in Fig. 1b), for (a) the FS and (b) the BS series. The slopes of the lines are estimates of $\mu_{2}$ in Eq. (6). Arbitrary units on the y-axis are used in order to display results from different gauges in the same graph.

white noise. A probable reason for this inconsistency in that, at scales larger than several days, the rainfall process does not exhibit a multiplicative structure. However, in the absence of a reliable physical interpretation, this metric may not be appropriate to study support intermittency in this scale range. The information provided by $\phi$ in the ranges of storms and frontal systems is similar to that given by $\mu_{2}^{\mathrm{BS}}$, as also demonstrated by the high correlation coefficients between these metrics $(>0.8)$. In the transition regime, the relation between $\phi$ and $\mu_{2}^{\mathrm{BS}}$ is instead weaker, confirming their poor efficacy when used at these large scales.

A last consideration can be derived through the comparison of $\mu_{2}^{\mathrm{FS}}$ and $\mu_{2}^{\mathrm{BS}}$, which allows quantifying the contribution of the support variability on overall intermittency. Table 2 shows that, in the storm regime, $\mu_{2}^{\mathrm{FS}}<\mu_{2}^{\mathrm{BS}}$, meaning that the variations of rainfall intensities smooth the support intermittency. In contrast, for scales larger than $32 \mathrm{~min}$, $\mu_{2}^{\mathrm{BS}}<\mu_{2}^{\mathrm{FS}}$, implying that the rainfall intensities have the effect of amplifying the support fluctuations, in accordance with results of Molini et al. (2009), who obtained similar values of the intermittency exponent in an analogous scale range.

\subsection{Linkage with synoptic circulation and topography}

As a next step of the study, we investigated the presence of spatial patterns for the metrics obtained in the two scaling ranges of frontal systems and storms. From this analysis, we excluded the transition regime because, in this range, results presented so far were often uncertain and characterized by unclear physical meaning, and also since these large scales have less relevance for hydrological applications. The spatial patterns for the metrics were obtained by applying the kriging technique on the whole island of Sardinia. Inspection of the maps reveals that meaningful spatial patterns only emerge in the regimes associated with frontal systems (shown in Fig. 7), while the metrics are more randomly distributed at scales typical of convective cells and single storms 

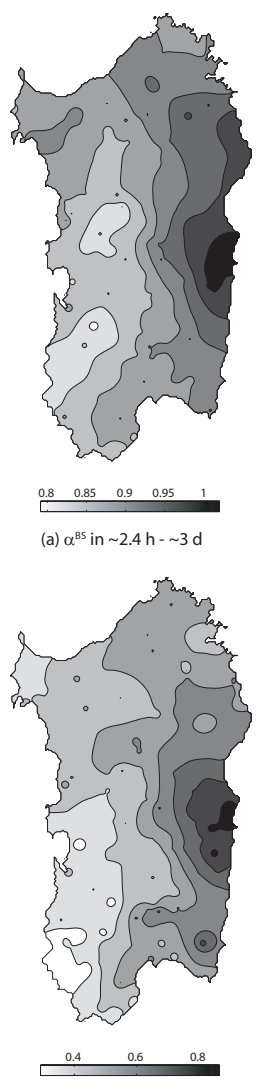

(d) $\alpha^{F 5}$ in $\sim 2.4 \mathrm{~h}-\sim 3 \mathrm{~d}$

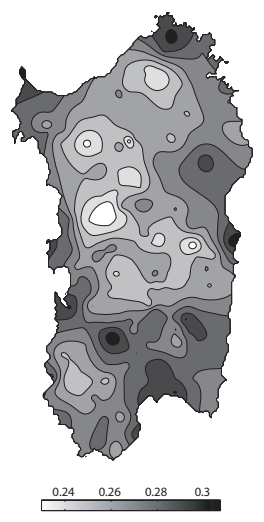

(b) $\phi$ on BS in 32 min $-\sim 3 \mathrm{~d}$

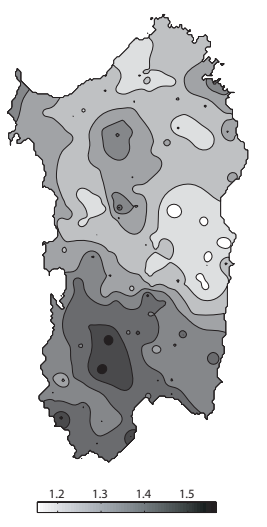

(e) $\mathrm{K}(3)$ on $\mathrm{FS}$ in $32 \mathrm{~min}-\sim 11 \mathrm{~d}$
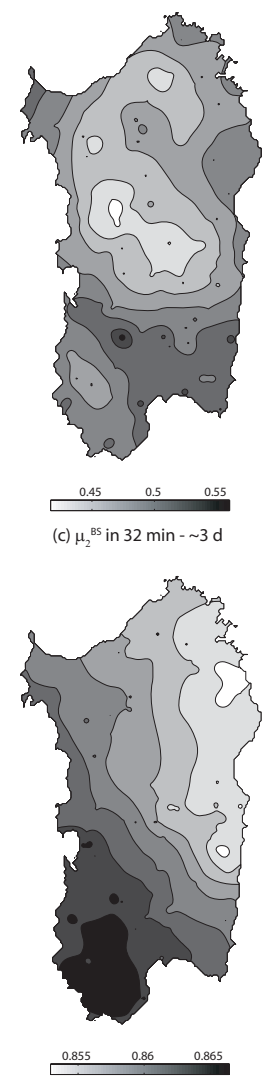

(f) $\mu_{2}^{\text {Fs }}$ in $32 \min -\sim 3 \mathrm{~d}$

Fig. 7. Spatial maps of the metrics in the range of frontal systems, obtained by (i) applying the kriging technique for the 201 gauges in a regular grid of $1 \mathrm{~km}$ (a power model has been fitted to the empirical variogram), and (ii) drawing the contour lines from the grid. Top (bottom) panels report results for the metrics applied to BS (FS) series.

( $<32 \mathrm{~min}$ ) (not shown). As a consequence, the connections with (i) the main rainfall regimes observed on the island and, in turn, with the dominant synoptic circulation as identified by Chessa et al. (1999), and (ii) the topographic features are only presented and discussed for the metrics obtained in the frontal system regime.

The metrics relative to $\mathrm{BS}\left(\alpha^{\mathrm{BS}}, \phi\right.$, and $\left.\mu_{2}^{\mathrm{BS}}\right)$ are reported in Fig. $7 \mathrm{a}-\mathrm{c}$, while those computed on FS $\left(\alpha^{\mathrm{FS}}, K(3)\right.$, and $\mu_{2}^{\mathrm{FS}}$ ) are shown in Fig. $7 \mathrm{~d}-\mathrm{f}$. The breaking points limiting the scale range in each case are also indicated in the subplot titles. The patterns of $\alpha^{\mathrm{BS}}$ and $\alpha^{\mathrm{FS}}$ are very similar (Fig. 7a and d). A significant correlation exists between $\phi$ and $\mu_{2}^{\mathrm{BS}}$ (Fig. 7b and c), even if the pattern of $\phi$ is more variable as compared to the smoother $\mu_{2}^{\mathrm{BS}}$, due to a stronger linkage with the elevation (described later). A similar consideration can also be made for $K(3)$ and $\mu_{2}^{\mathrm{FS}}$ (Fig. 7e and f), with $K$ (3) displaying more variability than $\mu_{2}^{\mathrm{FS}}$. Overall, the patterns of Fig. 7 resemble the clusters of the rainfall regimes of Fig. 2. The spectral exponents $\alpha^{\mathrm{BS}}$ and $\alpha^{\mathrm{FS}}$ (Fig. 7a and d)

are more correlated to the sirocco pattern (cluster 3), while the other metrics are more affected by the signature of mistral patterns, especially cluster 1 . The presence of linkages with synoptic circulation may be useful to improve the calibration of stochastic downscaling models that reproduce the rainfall time series at multiple scales, when these simulation tools are used in cascades to weather forecasting or climate prediction models.

As a subsequent analysis, we evaluated the linkages between the metrics and the topographic features at the gauge locations. For this purpose, we calculated the slope, aspect and elevation of each gauge using a DEM. While no specific relation was found between the metrics computed in any scaling regime and the slope or the aspect, a significant dependence was detected between the gauge elevation and the metrics calculated in the regime of frontal systems. This is shown in Fig. 8, where each plot refers to a metric and was built by (i) dividing the 201 gauges in 6 classes of elevation with approximately the same number of stations, and (ii) computing, for each class, the mean elevation of the gauges falling in that class, and the corresponding mean and standard deviation of the metric (plotted as circle and bars, respectively). The figures indicate that, as the elevation increases, (a) individual, more localized rainfall events are present within the $\mathrm{FS}$ and BS time series (increasing $\alpha^{\mathrm{BS}}$ and $\alpha^{\mathrm{FS}}$ ); (b) the rainfall statistical properties are characterized by decreasing rate of dissipation of the intensity fluctuations as the scale of aggregation decreases (decreasing $K(q)$ and $\mu_{2}^{\mathrm{FS}}$ ); and (c) the support clustering increases and the BS series are less intermittent (decreasing $\phi$ and $\mu_{2}^{\mathrm{BS}}$ ). The presence of a relation between intermittency properties of rainfall and elevation is important to develop regionalization techniques to refine parametrization of intensity-duration-frequency curves useful in land planning and design of civil infrastructures.

\subsection{Effect of the signal sampling methodology on intermittency analysis}

As already discussed in the Introduction, most of rainfall time series recorded by modern tipping-bucket rain gauges are provided according to the discrete counting (DC) approach (e.g., rainfall depth multiples of $0.2 \mathrm{~mm}$ with a 1 -min resolution). However, the use of DC may introduce a number of drawbacks that affect the intermittency analysis. To overcome these problems, in this study we proposed an alternative approach, the $\mathrm{CC}$ approach illustrated in Appendix A, which was applied to preprocess the signals used for all the analyses presented so far. In this subsection, we show evidence of how the use of DC can alter the values of the metrics adopted to study rainfall intermittency and, in turn, the physical interpretation. For this aim, all the metrics described in Sect. 4 were also calculated on the signals sampled at 1min resolution with the DC approach and compared against those obtained at the same resolution on the series built with the CC method. Prior to discussing results relative to each 

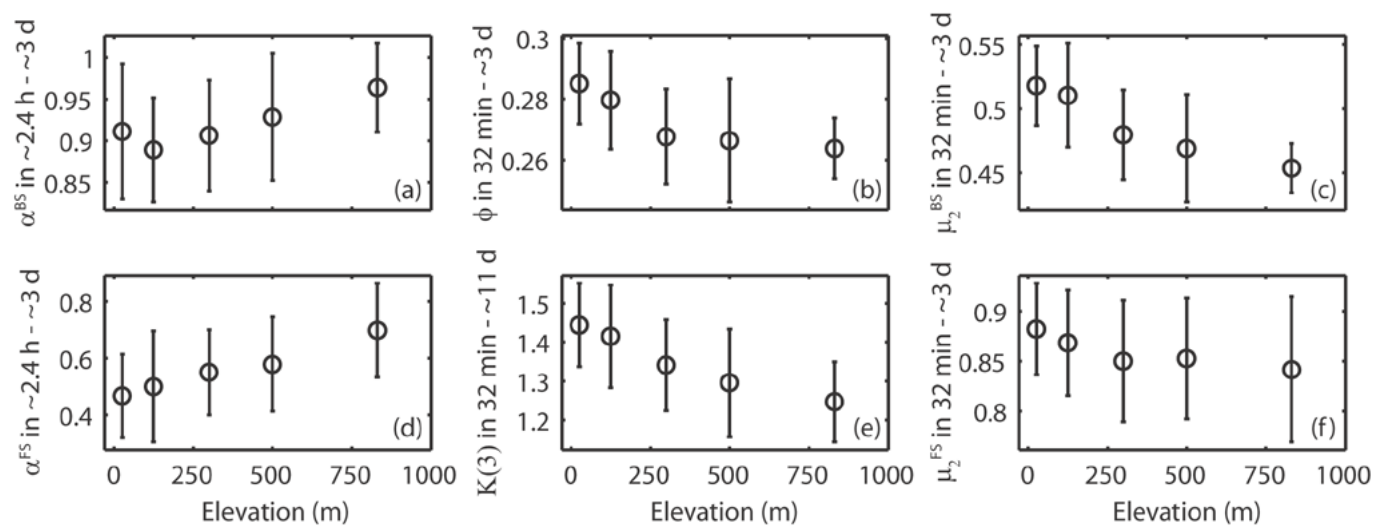

Fig. 8. Relation between the metrics in the range of frontal systems and the elevation; see main text for details.
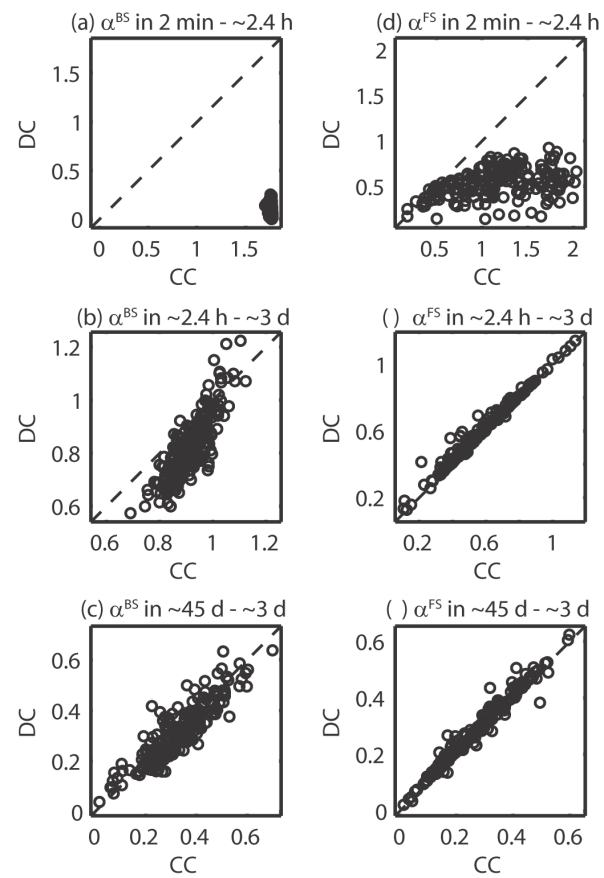

Fig. 9. Scatter plots between the spectral exponents computed for the signals sampled with $\mathrm{CC}$ (x-axis) and DC (y-axis) methods. Panels (a)-(c) and (d-f) refer to $\alpha^{\mathrm{BS}}\left(\alpha^{\mathrm{FS}}\right)$ for the three scaling regimes.

metric, we highlight that the same scaling regimes emerged from the signals created with both sampling approaches.

Figure 9 shows the scatter plots between the spectral exponents of BS and FS ( $\alpha^{\mathrm{BS}}$ and $\alpha^{\mathrm{FS}}$, respectively) derived from the series of all gauges sampled with CC (x-axis) and DC (yaxis) methods. The figures reveal that, at the highest frequencies between $2 \mathrm{~min}$ and $2.4 \mathrm{~h}$ (i.e., in the storm regime), significant differences emerge between the two methods (Fig. 9a and d). This is particularly evident for BS, where the mean $\alpha^{\mathrm{BS}}$ changes from 1.76 to 0.11 when considering CC and DC, respectively. Clearly, such a remarkable shift may induce wrong physical interpretation of the spectra. The differences between the $\alpha^{\mathrm{BS}}$ values are still present in the frontal regime and become minimal in the transition zone, with absence of any significant bias (Fig. 9b and c). Differences in $\alpha^{\mathrm{FS}}$ are instead negligible at scales larger than $\sim 2.4 \mathrm{~h}$ (Fig. 9e and f). Thus, distortions induced by the DC sampling strategy are more significant at smaller scales and for the binary signal.

Similar distortions as those affecting the spectral exponents $\alpha$ were also detected for the clustering $(\phi)$ and intermittency $\left(\mu_{2}\right)$ exponents. The scatter plots for the metric $\mu_{2}$ are shown in Fig. 10, where strong similarities with results of Fig. 9 are immediately apparent (e.g., a significant underestimation of $\mu_{2}^{\mathrm{BS}}$ in the DC case at scales lower than $32 \mathrm{~min}$ is evident from Fig. 10a). In addition, in this case significant biases are also present in the time regimes larger than $32 \mathrm{~min}$ (Fig. 10b, c, e, f). Note the opposite bias for the metrics $\mu_{2}^{\mathrm{BS}}$ and $\mu_{2}^{\mathrm{FS}}$ in the transition zone (Fig. 10c and f). Results for the clustering exponent $\phi$ are presented in Fig. 11 that, again, show evidence of similar distortions discussed for the previous figures.

Finally, comparison between results of the multifractal scale invariance analysis (Fig. 12) reveals that this metric is insensitive to the sampling method used to build the rainfall signal, as the multifractal exponents $K(3)$ calculated from the signal constructed with DC and CC approaches are always coincident. This is a significant result that should be kept in mind when selecting rainfall downscaling models.

\section{Conclusions}

We conducted a systematic study aimed at characterizing different aspects related to the nature of rainfall intermittency through the analysis of high-resolution (1-min) data recorded by a network of 201 tipping-bucket gauges covering the entire island of Sardinia (Italy). Four techniques including spectral and scale invariance analysis, and clustering and intermittency exponents, were used to investigate intermittency associated with (i) the alternation of dry and wet 

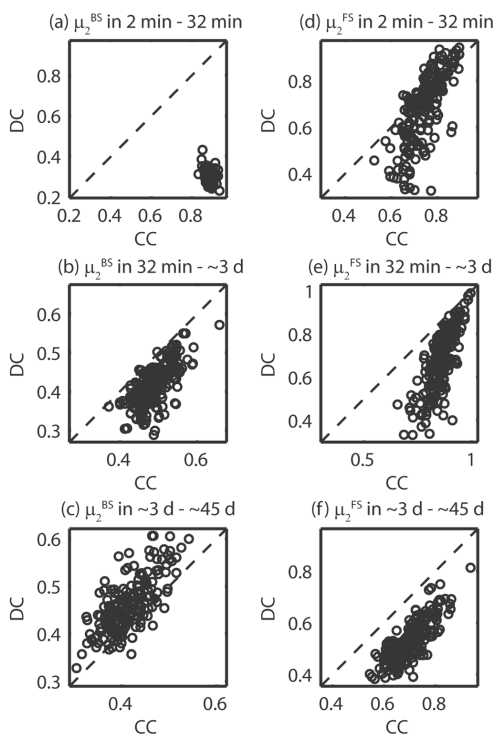

Fig. 10. Scatter plots between the intermittency exponents computed for the signal sampled with CC (x-axis) and DC (y-axis) methods. Panels (a)-(c) and (d-f) refer to $\mu_{2}^{\mathrm{BS}}\left(\mu_{2}^{\mathrm{FS}}\right)$ for the three scaling regimes.

periods (i.e., the variability of rainfall support) and (ii) the fluctuations of rainfall intensity amplitudes. Each of these tools involves the investigation of a scaling law and the computation of a scaling exponent, which was used as a metric to quantify the different aspects of rainfall intermittency.

Analyses revealed the presence of three scaling regimes, separated by breaking points whose positions change with the considered metric, most probably because such tools characterize different aspects of rainfall variability. Consistent with previous studies, the three scaling regimes can be associated with a transition zone at the largest scales ( $>\sim 3 \mathrm{~d}$ ), a regime typical of frontal systems at intermediate scales (between $\sim 2.4 \mathrm{~h}$ and $\sim 3 \mathrm{~d}$ ), and a range dominated by single storms at the smallest scales $(<\sim 2.4 \mathrm{~h})$. By analyzing the metric values, we obtained the following main results, which allow drawing some general conclusions:

1. The intermittency properties of rainfall support were found to be fairly similar across the analyzed region, while those of rainfall intensity are characterized by significant spatial variability. This implies that the local climate has a larger effect on the fluctuations of the rainfall amplitudes and minimal influence on the wet/dry process of rainfall occurrence.

2. The rainfall support is less clusterized in the storm regime as compared to the range of frontal systems.

3. The fluctuations of the rainfall amplitudes are similar across the aggregation scales in the storm regime, while they tend to increase at smaller time scales within the range of frontal systems.
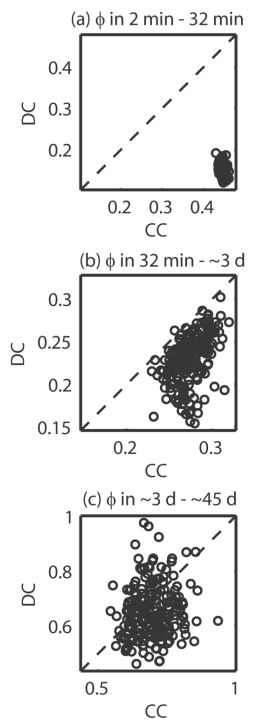

Fig. 11. Scatter plots between the clustering exponents $\phi$ computed for the signal sampled with $\mathrm{CC}$ (x-axis) and DC (y-axis) methods. Each panel refers to a given scaling regime.

4. In the transition regime, results are uncertain and cannot be readily supported by physical interpretations.

Thanks to the adequate spatial coverage of the gauge network, we also investigated the presence of spatial patterns for the metrics and found that these are only significant in the range of frontal systems. The patterns resemble those of the main pluviometric regimes and can thus be associated with the corresponding dominant synoptic circulation. These findings suggest the possibility to improve the calibration of rainfall downscaling models used in cascade to coarse predictions of climate or weather forecasting models. In addition, a relation was found between the metrics and the elevation of the gauges. This outcome may help the refinement of the parameterization of intensity-durationfrequency curves through regionalization techniques, useful in civil engineering applications.

Last but not least, we demonstrated how the discrete sampling methodology typically adopted to build the rainfall intensity signal for this type of modern tipping-bucket gauges leads to distortions in the analysis of rainfall intermittency. To limit the drawbacks, we suggested a method that allows sampling the signal in a more realistic fashion. Moreover, we showed that the multifractal scale invariance analysis is the only tool that is insensitive to the sampling approach and that the related metrics are neither biased nor distorted in any scaling regime. 

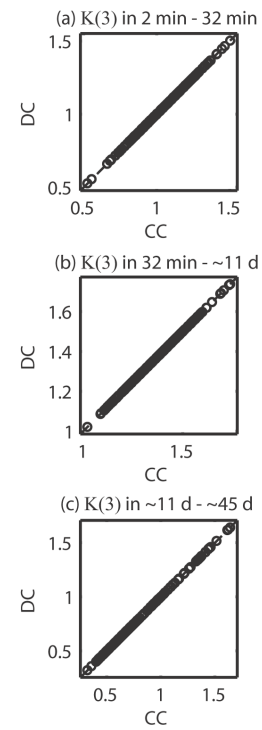

Fig. 12. Scatter plots between the multifractal exponents $K(3)$ computed for the signal sampled with $\mathrm{CC}$ (x-axis) and DC (y-axis) methods. Each panel refers to a given scaling regime.

\section{Appendix A}

\section{Signal sampling with DC and CC methods}

Let $i(t)$ and $H(t)$ (usually in $\mathrm{mm} \mathrm{h}^{-1}$ and $\mathrm{mm}$, respectively) be the continuous rainfall intensity and the cumulative rainfall depth functions, related as

$$
H(t)=\int_{0}^{t} i(\theta) \mathrm{d} \theta .
$$

If $H(t)$ is known, Eq. (A1) allows one to easily compute the mean rainfall intensity discretized at a given resolution $\Delta t$, by applying the derivative operator in discrete form:

$i_{\Delta t, k}=\frac{H\left(t_{k}+\Delta t\right)-H\left(t_{k}\right)}{\Delta t}$,

where $i_{\Delta t, k}$ is the mean rainfall intensity in the generic $k$-th time step $\Delta t$, included between the instants $t_{k}$ and $t_{k}+\Delta t$.

Let us assume that rainfall is measured by a tipping-bucket rain gauge with bucket volume that corresponds to $0.2 \mathrm{~mm}$ of rainfall. In the discrete counting (DC) sampling approach, the $0.2 \mathrm{~mm}$ of rain collected before a tip is recorded are assumed to instantaneously fall when the tip occurs. This implies that, if $N(t)$ is the number of tips that occurred up to a generic time $t$ and $r_{1}, r_{2}, \ldots, r_{N(t)}$ are the instants where the tips were recorded, the $H(t)$ function in the DC approach is defined as $H(t)=0.2 \cdot N(t) \mathrm{mm}$, which represents a step-wise line with steps of $0.2 \mathrm{~mm}$ located in $r_{1}, r_{2}, \ldots, r_{N(t)}$. This is illustrated in the qualitative example of Fig. A1a, where the crosses indicate the tipping instants and the black line is the
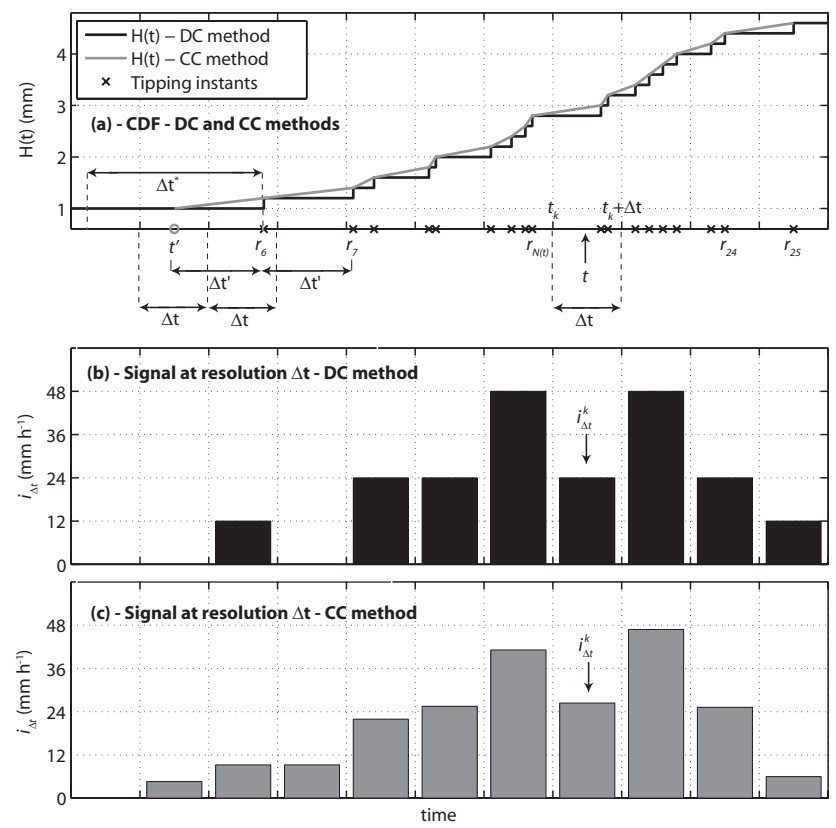

Fig. A1. Qualitative example illustrating the construction of the rainfall intensity signal with discrete counting (DC) and continuous counting (CC) methods. (a) Cumulative rainfall depth function $H(t)$ of the rainfall signal built with DC and CC methods: the symbols are described in Appendix A. (b) Rainfall intensity signal at resolution $\Delta t$ built with the DC method. (c) Same as (b) but for CC. The values reported on the $\mathrm{y}$-axis refer to the case of $\Delta t=1 \mathrm{~min}$ and an accumulated rainfall depth per tip of $0.2 \mathrm{~mm}$.

$H(t)$ function for the DC approach. Figure A1b shows the corresponding time series of rainfall intensities $i_{\Delta t}$ computed using Eq. (A2) and represented as a hyetograph.

The use of DC can lead to a signal with physical inconsistencies, including (i) isolated time steps with a relatively high value of $i_{\Delta t}$, and (ii) time steps with $i_{\Delta t}=0$ within rainfall events where it is likely raining without interruptions. Both shortcomings can be noticed at the beginning of the storm in Fig. A1b, where (i) the intensity in the first time step with $i_{\Delta t}>0$ is artificially set to a relatively high value, and (ii) there is a gap where $i_{\Delta t}=0$ between this time step and the bulk of the storm. Moreover, the signal is discretized (in our case, choosing a 1-min time step as suggested by WMO, the values of $i_{\Delta t}$ are multiples of $12 \mathrm{~mm} \mathrm{~h}^{-1}$ ).

To overcome the drawbacks of the DC approach, we used a strategy to sample the signal in continuous fashion (method labeled as CC, continuous counting). To construct the $H(t)$, we assume that the $0.2 \mathrm{~mm}$ recorded in a generic tip $r_{j}$ have been accumulated with constant intensity from $r_{j-1}$. In other words, we assume a linear interpolation between $H\left(r_{j-1}\right)$ to $H\left(r_{j}\right)$. This hypothesis requires dealing with an exception that occurs when we have consecutive tips that are too far away and, presumably, belong to different storms. In fact, if we linearly interpolate, we are assuming the presence of a constant rainfall of very low (or negligible) intensity 
between storms. To avoid this drawback, we introduce a time duration threshold $\Delta t^{*}$ such that, when $\left(r_{j}-r_{j-1}\right)>\Delta t^{*}$ (i.e., when tips are very distant), we assume that the $0.2 \mathrm{~mm}$ have been uniformly accumulated in the time interval from $t^{\prime}=\left(r_{j}-\Delta t^{\prime}\right)$ to $r_{j}$, where $\Delta t^{\prime}=\left(r_{j+1}-r_{j}\right)$. In other words, we hypothesize that the $0.2 \mathrm{~mm}$ of rainfall recorded at $r_{j}$ and at $r_{j+1}$ have fallen with same duration and intensity.

To better illustrate this, let us consider the instant $r_{6}$ in Fig. A1a. Here, the distance with the previous tip $r_{5}$ (not shown) is larger than $\Delta t^{*}$. To distribute the $0.2 \mathrm{~mm}$ of rainfall falling prior to $r_{6}$, we calculate the interval $\Delta t^{\prime}=\left(r_{7}-r_{6}\right)$ and we assume that the $0.2 \mathrm{~mm}$ have been uniformly accumulated between $t^{\prime}=\left(r_{6}-\Delta t^{\prime}\right)$ and $r_{6}$. As a result, we introduce a time $t^{\prime}$ where the storm begins (gray circle in Fig. A1a). From a physical point of view, the duration $\Delta t^{*}$ is associated with the typical cell life duration, which can range from ten minutes to more than half an hour. We tested several $\Delta t^{*}$ values within this range, obtaining similar results in terms of scaling regimes and metrics. In this work, we assumed $\Delta t^{*}=15 \mathrm{~min}$ as this is physically consistent with the rainfall characteristics in the study area.

The $H(t)$ function built with the CC approach is shown as a gray line in Fig. A1a, while the corresponding time series $i_{\Delta t}$, computed with Eq. (A2), is shown in Fig. A1c. Note how, in this case, the rainfall intensities at the beginning of the storm appear more realistic and the whole signal is not anymore discretized. We highlight that, while we analyzed the time series of our rain gauges, we found cases of single isolated tips, very distant from antecedent and subsequent rainfall events. In these situations, it is very likely that no precipitation has effectively fallen and that the presence of a tip may be due to dew or other problems of the recording apparatus. As a result, we decided to remove these isolated tips before building the signal. This has led to negligible differences in the total accumulated rainfall.

\section{Appendix B}

\section{Detection of breaking points}

The application of the four techniques led to the identification of the three ranges of scaling regimes where the power laws Eqs. (1), (3), (4), and (6) hold with different exponents. In the following, we illustrate the procedure to detect the breaking points that separate the scaling regimes for the case of scale invariance (Eq. 3). The approach, adapted with the proper change of variable names, was also applied to study the scaling regimes of the other analysis tools used in the paper. The position of the first breaking point $t_{\mathrm{BP}, 1}$ was searched by (i) assuming $t_{\mathrm{BP}, 1}=2^{j}$, with $j=2$ in the first iteration; (ii) applying the linear regression in the range between $\log \left(\tau_{0}\right)$ and $\log \left(t_{\mathrm{BP}, 1}\right)$; and (iii) computing the rootmean-square error (RMSE) between points and regression line. Steps (i)-(iii) were repeated for increasing values of $j$ up to a maximum value $j_{\max }$ that we fixed based on visual inspection of the relations $\log \left[S_{q}(\tau)\right]$ versus $\log (\tau)$ of all stations. The first breaking point was set as $t_{\mathrm{BP}, 1}=2^{j^{*}}$, where $j^{*}\left(2 \leq j^{*} \leq j_{\max }\right)$ is the index for which the RMSE is minimum. The same procedure was then repeated to determine $t_{\mathrm{BP}, 2}$, starting from $t_{\mathrm{BP}, 1}$.

Acknowledgements. The authors thank two anonymous reviewers, whose comments significantly helped to improve the quality of the manuscript. The authors also acknowledge the Regione Autonoma della Sardegna (grant F71J09000120002, L.R. n. 7/2007) and the Agenzia Regionale del Distretto Idrografico della Sardegna (cooperation agreement).

Edited by: A. A. Carsteanu

\section{References}

Badas, M. G., Deidda, R., and Piga, E.: Modulation of homogeneous space-time rainfall cascades to account for orographic influences, Nat. Hazards Earth Syst. Sci., 6, 427-437, doi:10.5194/nhess-6-427-2006, 2006.

Bershadskii, A. J. N., Praskovsky, A., and Sreenivasan, K.: Clusterization and intermittency of temperature fluctuations in turbulent convection, Phys. Rev., doi:10.1103/PhysRevE.69.056314, 2004.

Burton, A., Fowler, H., Blenkinsop, S., and Kilsby, C.: Downscaling transient climate change using a Neyman Scott Rectangular Pulses stochastic rainfall model, J. Hydrology, 381, 18-32, doi:10.1016/j.jhydrol.2009.10.031, 2010.

Chessa, P. A., Cesari, D., and Delitala, A. M. S.: Mesoscale Precipitation and Temperature Regimes in Sardinia (Italy) and their Related Synoptic Circulation, Theor. Appl. Climatol., 63, 195221, doi:10.1007/s007040050103, 1999.

Chessa, P. A., Ficca, G., Marrocu, M., and Buizza, R.: Application of a Limited-Area Short-Range Ensemble Forecast System to a Case of Heavy Rainfall in the Mediterranean Region, Weather Forecast., 19, 566-581, 2004.

Cuo, L., Pagano, T. C., and Wang, Q. J.: A Review of Quantitative Precipitation Forecasts and Their Use in Short- to MediumRange Streamflow Forecasting, J. Hydrometeorol., 12, 713-728, doi:10.1175/2011JHM1347.1, 2011.

de Lima, M. and Grasman, J.: Multifractal analysis of 15-min and daily rainfall from a semi-arid region in Portugal, J. Hydrol., 220, 1-11, doi:10.1016/S0022-1694(99)00053-0, 1999.

Deidda, R.: Rainfall downscaling in a space-time multifractal framework, Water Resour. Res., 36, 1779-1794, 2000.

Deidda, R., Benzi, R., and Siccardi, F.: Multifractal modeling of anomalous scaling laws in rainfall, Water Resour. Res., 35, 18531867, 1999.

Deidda, R., Badas, M. G., and Piga, E.: Space-time scaling in highintensity Tropical Ocean Global Atmosphere Coupled OceanAtmosphere Response Experiment TOGA-COARE storms, Water Resour. Res., 40, W02506, doi:10.1029/2003WR002574, 2004.

Deidda, R., Badas, M. G., and Piga, E.: Space-time Multifractality of Remotely Sensed Rainfall Fields, J. Hydrol., 322, 2-13, doi:10.1016/j.jhydrol.2005.02.036, 2006. 
Dingman, S. L.: Physical Hydrology, Waveland Press, Inc., 2008.

Eagleson, P. S.: Ecohydrology: Darwinian Expression of Vegetation Form and Function, 2002.

Fraedrich, K. and Larnder, C.: Scaling regimes of composite rainfall time series, Tellus, 45, 289-298, 1993.

Georgakakos, K. P. and Kavvas, M. L.: Precipitation analysis, modeling, and prediction in hydrology, Rev. Geophys., 25, 163-178, 1987.

Guadagnini, A. and Neuman, S.: Extendend power-law scaling of self affine signals exhibiting apparent multifractality, Geophys. Res. Lett., 38, L13403, doi:10.1029/2011GL047727, 2011.

Harris, D., Seed, A., Menabde, M., and Austin, G.: Factors affecting multiscaling analysis of rainfall time series, Nonlin. Processes Geophys., 4, 137-156, doi:10.5194/npg-4-137-1997, 1997.

Huffman, G. J., Adler, R. F., Arkin, P., Chang, A., Ferraro, R., Gruber, A., Janowiak, J., McNab, A., Rudolf, B., and Schneider, U.: The Global Precipitation Climatology Project (GPCP) Combined Precipitation Dataset, B. Am. Meteorol. Soc., 78, 5-20, doi:10.1175/1520-0477(1997)078;0005:TGPCPG;2.0.CO;2, 1997.

Kundu, P. K. and Siddani, R. K.: Scale dependence of spatiotemporal intermittence of rain, Water Resour. Res., 47, W08522, doi:10.1029/2010WR010070, 2011.

Langousis, A. and Veneziano, D.: Intensity-Duration-Frequency Curves from Scaling Representations of Rainfall, Water Resour. Res., 43, W02422, doi:10.1029/2006WR005245, 2007.

Langousis, A. and Veneziano, D.: Theoretical Model of Rainfall in Tropical Cyclones for the Assessment of Long-term Risk, J. Geophys. Res., 114, D02106, doi:10.1029/2008JD010080, 2009a.

Langousis, A. and Veneziano, D.: Long-term Rainfall Risk from Tropical Cyclones in Coastal Areas, Water Resour. Res., 45, W11430, doi:10.1029/2008WR007624, 2009b.

Langousis, A., Veneziano, D., Furcolo, P., and Lepore, C.: Multifractal Rainfall Extremes: Theoretical Analysis and Practical Estimation, Chaos Soliton Fract., 39, 1182-1194, doi:10.1016/j.chaos.2007.06.004, 2009.

Lanza, L., Leroy, M., Alexandropoulos, C., Stagi, L., and Wauben, W.: WMO Laboratory intercomparison of rainfall intensity gauges, Tech. rep., World Meteorological Organization, 2005.

Lovejoy, S. and Schertzer, D.: Generalized scale invariance and fractal models of rain, Water Resour. Res., 21, 1233-1250, 1985.

Lovejoy, S. and Schertzer, D.: Multifractals, universality classes, and satellite and radar measurements of cloud and rain fields, J. Geophys. Res., 95, 2021-2031, multifrattali della pioggia, 1990.

Mascaro, G., Vivoni, E. R., and Deidda, R.: Implications of Ensemble Quantitative Precipitation Forecast Errors on Distributed Streamflow Forecasting, J. Hydrometeorol., 11, 69-86, 2010.

Molini, A., Katul, G. G., and Porporato, A.: Revisiting rainfall clustrering and intermittency across different climatic regimes, Water Resour. Res., 45, doi:10.1029/2008WR007352, 2009.

Moonen, A. C., Ercoli, L., Mariotti, M., and Masoni, A.: Climate change in Italy indicated by agrometeorological indices over 122 years, Agr. Forest Meteorol., 111, 13-27, 2002.

Neuman, S.: Apparent/spurious multifractality of data sampled from fractional Brownian/Levy motions, Hydrol. Process., 24, 2056-2067, doi:10.1002/hyp.7611, 2010a.

Neuman, S.: Apparent/spurious multifractality of absolute increments sampled from truncated fractional Gaussian/Levy noise, Geophys. Res. Lett., 37, L09403, doi:10.1029/2010GL043314,
$2010 b$.

Neuman, S.: Apparent multifractality and scale-dependent distribution of data sampled from self-affine processes, Hydrol. Process., 25, 1837-1840, doi:10.1002/hyp.7967, 2012.

Onof, C. and Wheater, H. S.: Modelling of British rainfall using a random parameter Bartlett-Lewis rectangular pulse model, J. Hydrol., 149, 67-95, 1993.

Purdy, J. C., Harris, D., Austin, G. L., Seed, A. W., and Gray, W.: A case study of orographic rainfall processes incorporating multiscaling characterization techniques, J. Geophys. Res., 106, 7837-7845, 2001.

Rebora, N., Ferraris, L., von Hardenberg, J., and Provenzale, A.: RainFARM: Rainfall Downscaling by a Filtered Autoregressive Model, J. Hydrometeorol., 7, 724-738, doi:10.1175/JHM517.1, 2006.

Rigby, J. and Porporato, A.: Precipitation, dynamical intermittency, and sporadic randomness, Adv. Water Resour., 33, 923-932, doi:10.1016/j.advwatres.2010.04.008, 2010.

Robinson, J. S. and Sivapalan, M.: Temporal scales and hydrological regimes: Implications for flood frequency scaling, Water Resour. Res., 33, 2981-2999, doi:10.1029/97WR01964, 1997.

Rodriguez-Iturbe, I., Cox, D. R., and Isham, V.: Some Models for Rainfall Based on Stochastic Point Processes, Proc. R. Soc. Lon. Ser.-A, 410, 269-288, 1987.

Schleiss, M., Jaffrain, J., and Berne, A.: Statistical analysis of rainfall intermittency at small spatial and temporal scales, Geophys. Res. Lett., 38, doi:10.1029/2011GL049000, 2011.

Seo, D.-J., Perica, S., Welles, E., and Schaake, J. C.: Simulation of precipitation fields from probabilistic quantitative precipitation forecasts, J. Hydrol., 239, 203-229, 2000.

Sreenivasan, K. R. and Bershadskii, S.: Clustering Properties in Turbolent Signals, J. Stat. Phys., 125, 1145-1157, 2006.

Trenberth, K. E., Dai, A., Rasmussen, R. M., and Parsons, D. B.: The Changing Character of Precipitation, B. Am. Meteorol. Soc., 84, 1205-1217, doi:10.1175/BAMS-84-9-1205, 2003.

Veneziano, D. and Furcolo, P.: Improved moment scaling estimation for multifractal signals, Nonlin. Processes Geophys., 16, 641653, doi:10.5194/npg-16-641-2009, 2009.

Veneziano, D. and Iacobellis, V.: Self-similarity and multifractality of topographic surfaces at basin and subbasin scales, J. Geophys. Res., 104, 12797-12812, doi:10.1029/1999JB900083, 1999.

Veneziano, D. and Langousis, A.: The Areal Reduction Factor: a Multifractal Analysis, Water Resour. Res., 41, W07008, doi:10.1029/2004WR003765, 2005a.

Veneziano, D. and Langousis, A.: The Maximum of Multifractal Cascades: Exact Distribution and Approximations, Fractals, 13, 311-324, 2005b.

Veneziano, D., Bras, R. L., and Niemann, J. D.: Nonlinearity and self-similarity of rainfall in time and a stochastic model, J. Geophys. Res., 101, 26371-26392, doi:10.1029/96JD01658, 1996.

Veneziano, D., Langousis, A., and Furcolo, P.: Multifractality and Rainfall Extremes: A Review, Water Resour. Res., 42, W06D15, doi:10.1029/2005WR004716, 2006.

Veneziano, D., Lepore, C., Langousis, A., and Furcolo, P.: Marginal Methods of Intensity-duration-frequency Estimation in Scaling and Nonscaling Rainfall, Water Resour. Res., 43, W10418, doi:10.1029/2007WR006040, 2007.

Venugopal, V., Foufula-Georgiou, E., and Sapozhnikov, V.: Evidence of dynamic scaling in space-time rainfall, J. Geophys. 
Res., 104, 31599-31610, 1999.

Venugopal, V., Roux, S. G., Foufoula-Georgiou, E., and Arneodo, A.: Revisiting multifractality of high-resolution temporal rainfall using a wavelet-based formalism, Water Resour. Res., 42, doi:10.1029/2005WR004489, 2006.
Verrier, S., Mallet, C., and Barthés, L.: Multiscaling properties of rain in the time domain, taking into account rain support biases, J. Geophys. Res., 116, doi:10.1029/2011JD015719, 2011. 